\title{
ESTABLECIMIENTO DE ESPECIFICACIONES PARA UN ESTANDAR E-LEARNING EN LA UNIVERSIDAD DEL QUINDÍO
}

\author{
SETTING A SPECIFICATIONS FOR E-LEARNING STANDARD \\ AT THE UNIVERSIDAD DEL QUINDÍO
}

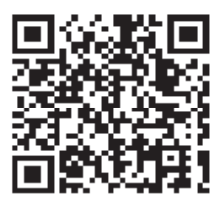

Edgar Javier Carmona Suárez ${ }^{1}$,Elizabeth Rodríguez Salinas ${ }^{2}$

${ }^{1 .}$ Profesor titular en la Universidad del Quindío, Grupo de investigación GEDES.

2. Profesora Universidad Central de Colombia, Grupo de investigación AVEA.

Recibido: Noviembre de 2015

Aceptado: Julio de 2016

*Correspondencia del autor: Edgar Javier Carmona Suárez; E-mail: carmonaedgar@hotmail.com.

\begin{abstract}
RESUMEN
El aumento en la oferta de cursos virtuales es solución para varios sectores de la población, dado que permite estudiar de manera asincrónica lo cual significa que no tendrá que asistir a ningún lugar para recibir la capacitación, rompiendo así las barreras de espacio y tiempo, cada estudiante aprende a su propio ritmo con soporte y control de un profesor capacitado para ello. El crecimiento en la oferta virtual, en muchas instituciones surge de iniciativas individuales, es desordenado y en ocasiones caótico, lo que exige centrar la atención en nuevos problemas. Uno de ellos es la falta de unidad de criterio de los espacios académicos en una misma universidad o incluso en un mismo programa. Esta investigación se propuso inicialmente hacer un estudio de cursos virtuales de la Universidad del Quindío y, a la luz de estándares internacionales en E-learning, generar un conjunto de especificaciones que propicien la normalización y estandarización adaptadas a las necesidades regionales, para ello se utilizó la técnica de investigación etnográfica y de Investigación Acción Participación (IAP). Se centró la atención en los aspectos técnicos, comunicativos, administrativos y pedagógicos de la producción de cursos virtuales.
\end{abstract}

Palabras claves: Estándares, educación virtual,E-learning, calidad educativa.

\begin{abstract}
The increase in the supply of online courses is solution for a given sector of the population breakthroughs barriers of space and time, individual training and learning different rhythms. This growth comes in many institutions of individual initiatives; it is sometimes messy and chaotic, which requires focusing on new problems. One is the lack of uniform standards of academic spaces in the same university or even in the same program. This research was initially proposed a study of online courses at the University of Quindío and in the light of international standards in E-learning, generate a set of specifications that promote the normalization and standardization adapted to regional.
\end{abstract}

Keywords: Standards, virtual education, E-learning, educational quality online courses. 


\section{INTRODUCCIÓN}

En Colombia se aprecia una creciente oferta en la educación virtual; a tal punto que todas las universidades ofrecen cursos virtuales (Facundo, 2011), (Carrasco \& González, 2011) y (Sierra, 2009). La Universidad del Quindío también ha ido aumentandopaulatinamente la oferta de cursos virtuales. Pero, en este crecimiento no ha mediado un proceso de planeación sistemático y ordenado, sino que ha obedecido a iniciativas de docentes (Carmona y Berrio, 2012 y Carmona 2013). Se hace necesario entonces emprender varias acciones, que orienten, ya no a la implementación de cursos y programas virtuales, sino al mejoramiento de la educación, utilizando las potencialidades de las TIC. La normalización y estandarización son una buena alternativa para iniciar procesos que conduzcan a garantizar programas virtuales de calidad, en atención a que uno de los factores más importantes para que el material instructivo sea de calidad, es que sea útil para el logro de aprendizajes, bajo normas y estándares mínimos (Morales, 2014).

En el contexto internacional, se observa como en la gran mayoría de las instituciones, y tal como lo afirma Hilera y Hoya (2010), toda esta proliferación de cursos virtuales surgieron y se desarrollaron sin un marco adecuado en cuanto a metodologías técnicas, documentales y psicopedagógicas, lo que conduce a problemas de accesibilidad, interoperabilidad, durabilidad, interactividad y reutilización de los materiales curriculares, disponibles en las diferentes redes de comunicación(Hilera \& Hoya, 2010).

Para Varela (2015) una forma de aproximarse al concepto de calidad en la formación virtual es a través de las certificaciones que ofrecen las agencias de Normalización, evaluación y certificación, tales como AENOR, en España o CALED, en Ecuador. Se precisa entonces empezar por la utilización de procesos de normalización, que orientenla calidad de los materiales educativos. Enfrentar este problemaconduce al concepto de "estándar".

Según la International Organization for Standarization (ISO), que reúne las diferentes organizaciones nacionales de estandarización: "La estandarización es la actividad que tiene por objeto establecer, ante problemas reales o potenciales, disposiciones destinadas a usos comunes y repetidos, con el fin de obtener un nivel de ordenamiento óptimo en un contexto dado, que puede ser tecnológico, político o económico"(ISO, 2009).

Estos estándares, en algunos casos, son de obligado cumplimiento, tal es el caso de páginas web que deben cumplir un determinado nivel de accesibilidad para discapacitados. Los estándares de facto son losque se usan por voluntad propia o conveniencia y tienen una amplia aceptación, aunque no hayan sido sancionados por un organismo de estandarización. El caso más conocido en Internet, son las recomendaciones realizadas por el World Wide Web Consortium (W3C), que crea las normas más utilizadas en la red, como por ejemploel lenguaje HTML.

\section{METODOLOGÍA}

El objetivo principal de la investigación fue formular un conjunto de especificaciones que orienten el establecimiento de un estándar E-learning, de manera que facilite la normalización de los cursos virtuales en la Universidad del Quindío. Esta investigación tiene una orientación cualitativa, ya que combina técnicas de investigación etnográfica y de Investigación Acción Participación (IAP). El componente etnográfico se fundamenta en la reflexión en torno al estudio de la evolución de la virtualidad en la Universidad, al análisis del estado del arte de los estándares en E-learning y a la forma como los artefactos tecnológicos median dinámicas educativas. Tiene un componente de investigación-acción participación dado la condición de investigadores inmersos en el objeto de estudio, que buscan el mejoramiento colectivo, mediante la creación de un conjunto de especificaciones que incidirán directamente en las políticas de educación virtual en la Institución.

Se hizo una revisión bibliográfica de los estándares para E-learning lo que permitió la fundamentación teórica; luego se hizo una observación activa, mediante la visita a cinco universidades, con el fin de hacer una contextualización nacional sobre el estado del arte. Al interior de la Universidad se realizaron entrevistas no estructuradas a líderes de estrategias virtuales y encuestas a docentes orientadores de cursos virtuales. Paralelamente se aplicaron instrumentos de observación a una muestra de los cursos en la plataforma educativa para analizar el estado del E-learning en la Universidad. De igual manera se diseñaron y aplicaron instrumentos y métodos de observación a una selección de cuarenta docentes de la Universidad. 
Finalmente, se elaboraron las especificaciones, las cualesse consignan en un instrumento que sirve de guía a las instituciones en el diseño de cursos virtuales, y al docente para que pueda, demanera más asertiva, enfrentarse a procesos de virtualización.

\section{DISCUSIÓN Y RESULTADOS}

En la práctica pedagógica mediada por las TIC el uso de estándares E-learning evita diversos problemas, entre ellos: el de portabilidad, es decir, que los contenidos se puedan mover y almacenar en diferentes plataformas; la interactividad, relacionada con el alto grado de participación e intercambio de experiencias entre los participantes; la interoperabilidad, esto es, que funcionen en distintas plataformas de hardware y software, sean reutilizables, sirvan como base para la creación de otros cursos y aplicaciones, sean accesibles y usables, que tengan metadatos para facilitar su localización. Hasta el momento, no existe una metodología única que garantice los objetivos de accesibilidad, interoperatividad, usabilidad, accesibilidad y reutilización de los materiales educativos en la Web (Hilera \& Hoya, 2010) e (Hilera, 2015).

Varias organizaciones han generado estándares Elearning, entre las que se pueden citar: ADL ( $\mathrm{La}$ Advanced Distributed Learning), AICC (Aviation Industry Computer Based Trainig Comitte o Comité de la Industria de la Aviación para el aprendizaje basado en Computadoras), CEN (Comité Europeo de Normalización), IEEE (Institute of Electrical and Electronic Engineers, y dentro de él, el LTSC o Comité de Estandarización de las Tecnologías Aplicadas al Aprendizaje), IMS GLC (IMS Global Learning Consortium), ISO/IEC (International Standards Organisation en colaboración con International Electrotechnical Commission) ARIADNE (Alliance of Remote Instructiona Authoring and distribution Network forEurope), DCMI (Dublin Core Metadata Intiative) y W3C (World Wide Web Consortium). Estas organizaciones, han creado estándares que se encargan de normalizar el E-learning categorizado en: contenidos y evaluaciones, usabilidad, accesibilidad, interoperatividad, arquitectura, calidad, competencias, derechos de autor, información del estudiante, metadatos, procesos de aprendizaje, repositorios, vocabulario y lenguajes. Algunos de ellos son: IMS DALA (2005), SCORM CAM (2006), UNIQUe (2006), ISO/IEC 24751-1 (2008), ISO 9001 (2008), LORN MD (2008) y WAI ARIA (2009).

En Colombia, en 2005, el Ministerio de Educación Nacional (M.E.N.), lanzó el primer Concurso Nacional de Objetos de Aprendizaje, con el propósito de consolidar por primera vez un banco de objetos de aprendizaje y ponerlo a disposición de la comunidad educativa nacional e internacional. Uno de los requerimientos para los participantes consistió en crear una ficha técnica con todos los atributos y descripción de uso del objeto de aprendizaje, de tal forma, que permitiera una fácil catalogación e intercambio de los mismos. Lo anterior, obligó a pensar en la búsqueda, análisis y revisión de estándares existentes. El M.E.N. adoptó entonces, el metadato Dublin Core, el empaquetamiento mediante SCORM, el sistema de administración de contenidos (CMS) DRUPALy D Space para conformar el Banco de Objetos (Ministerio de Educación Nacional, 2012).

Un año más tarde, fue necesario hacer visible y utilizables, los objetos de aprendizaje creados, para lo cual, se pensó en el M.E.N. en un estándar de clasificación fácil y oportuna, para el acceso a los objetos, los cuales estuvieran dispuestos en categorías y áreas del conocimiento. Se definió entonces un perfil de aplicación de un estándar de metadatos, basado en el estándar IEEE LOM y ajustando el perfil de aplicación a los requerimientos propios del país, se implementó un estándar que se denominó LOM CO, para describir los aspectos técnicos y educativos de los Objetos del Banco Nacional de Objetos y de los Bancos Institucionales.LOM CO define los siguientes metadatos obligatorios para los Objetos de Aprendizaje del Banco Nacional y considera opcionales, los correspondientes al resto de campos de la especificación IEEE LOM (Agudelo Benjumea, 2009):

a) General: título, idioma, descripción y palabras clave.

b) Ciclo de Vida: versión, autor(es), entidad $y$ fecha.

c) Técnico: formato, tamaño, ubicación, re querimientos e instrucciones de uso.

d) Educacional: tipo de interactividad, tipo de recurso de aprendizaje, nivel de interactividad, población objeto y contex to de aprendizaje.

e) Derechos: costo, derechos de autor y otras restricciones.

f) Relación: define la relación entre objetos 
de aprendizaje.

g) Anotación: uso educativo.

h) Clasificación: fuente de clasificación y ruta taxonómica.

El gobierno colombiano tomó conciencia acerca de la importancia de la educación virtual y por ello promulgó el Decreto 1295 de 2010, por medio del cual dotó a la educación de herramientas para desarrollar una nueva filosofía educacional, y es hacia allá donde debe apuntar la tarea del docente: sin ella no es posible alcanzar altos estándares de calidad, suficientes para entregar profesionales probos al sector laboral del país (Zuluaga, 2010 y Morales 2014).

En esta investigación, después del análisis de los estándares y especificaciones utilizados en la enseñanaza, y mundialmente reconocidos por los organismos citados, se encontró que existe una amplia gama estándares, por lo que fue necesario limitarel análisis solo a algunos. De igual manera,se detectó que ningún estandar cubre todos los aspectos que los contextos universitarios demandan. Aquí coincidimos con el informe 16 del Ministerio de Educación y Ciencia de España, en el cual se les denomina como "los más maduros" y son aquellos que abordan aspectos relativos a los contenidos, cómo se empaquetan los cursos, cómo se describen tanto los cursos como los propios elementos que componen dichos cursos (Fernández, Moreno, Sierra, \& Martínez, 2008).

Se consideró así, que tres (3) experiencias son suficientemente argumentadas, como para ser consideradas en procesos de estandarización en cualquier institucion universitaria,son ellas:IMS (Global Learning Consortium, Inc.) es el principal promotor y desarrollador de especificaciones abiertas, y cubren más aspectos de la educacion virtual; el desarrollado por ADL en su modelo de referencia SCORM, y, el aporte de IEEE LTSC con su propuesta de metadatos para objetos de aprendizaje.

De igual manera se planteó elinterrogante: ¿Es posible diseñar un conjunto de especificaciones que permitan la creación de un estándarE-learning para la universidad del Quindío? En el intento por responder a esta pregunta, los investigadores dieñan estrategias de implementación tecnológica, que impacten positivamente los espacios de aprendizaje al interior de la Universidad.
Si bien los estándares están construidos para cumplir normas, en este ejercicio de reflexión, los investigadores han comprendido la necesidad de acomodar las especificaciones generalizantes a situaciones contextuales; entendiendo que en la compleja realidad educativa, interactúan variedad de factores que estimulan o interfieren los procesos de aprendizaje. Es claro que el seguimiento de una política internacional permitiría a la Universidad del Quindío una normalización mundialmente reconocida y avalada, pero teambién es necesario responder con propuestas a contextualizadas a las necesidades de la región.

\section{Propuesta de especificaciones para los estándares de educación virtual}

Varios autores coinciden en la necesidad de considerar las categorías que fundamentan los requerimientos del estándar (Viscay, 2005) (Hilera González \& Hoya Marin, 2010).

La norma UNE 66181:2012, establece los parámetros de la calidad para la formaciónvirtual y de la gestión de la calidad, haciendo referencia a la empleabilidad, el nivel dereutilización, la metodología, etc. Para CALED, la calidad en la formación virtual estárelacionada con cuatro aspectos: tecnología, formación, diseño instruccional y serviciosy soporte (Varela, 2015).

Para efectos de la Universidad del Quindío, se consideró importante la definición de las siguientes características:

- Accesibilidad, en cuanto aque sea accesible para todas las personas de acuerdo (Hilera, 2013) e (Hilera, 2013).

- Transversalidad: los contenidos educativos deben abordar temas generales y deben tener la capacidad de ser usados en diversas áreas del conocimiento (Ética, Emprendimiento, Proficiencias, Constitución Política, etc.).

- Transmedia: los contenidos educativos deben prepararse, diseñarse y producirse, aprovechando la peculiaridad del medio. Esta característica está relacionada con la "granularidad", que hace referencia al aprovechamiento narrativo del medio que lo soporta y, da la posibilidad de articularse a estructuras globales (cursos).

- Diseño funcional: cada contenido educativo debe responder a un propósito y a una intención educativa. Los docentes han de trabajar de manera articulada, con equipos creativos, expertos en diseño, 
formas narrativas, animación y composición, para orientar de la mejor manera la producción de los contenidos educativos.

- Gestión administrativa: cada contenido debe atender a una necesidad académica que fundamente, soporte y oriente el proceso de producción.

- Licenciamiento: es preciso establecer términos y condiciones claras para el uso, modificación y personalización de los contenidos educativos, $y$, en lo posible, la promoción del Copy left, a través de las licencias Creative Commons (En particular Creative Commons Colombia), sin desconocer los sistemas tradicionales de licenciamiento.

- Unidad conceptual: la institución debe establecer un marco conceptual para el entendimiento y difusión del proyecto educativo institucional.

Igualmente, para presentar la propuesta de estándar para los cursos mediados con tecnología, se consideró importante y fundamental retomar los conceptos establecidos en el Marco Conceptual de la Estrategia Virtual de la Universidad del Quindío, los cuales definen las metodologías, dentro de las que se desarrollan cursos E-learning y b-learning.

\section{Concepto de estándar para los cursos B-learning y E-learning}

tadas en la realidad educativa y el contexto local. Los criterios de respeto por la propiedad intelectual, así como los aspectos administrativos, son algunos de los componentes a tener en cuenta en la implementación de estrategias tecnológicas dentro del Proyecto Educativo Institucional (PEI) de la Universidad del Quindío. A continuación se presentan las especificaciones que componen, según esta propuesta, el estándar de calidad E-learning y B-learning para la Universidad del Quindío. Dichas especificaciones han de tenerse en cuenta al diseñar y planear recursos educativos.

\section{ESPECIFICACIONES}

Cualidad que busca garantizar que el curso o recursopueda ser consultado y/o utilizado por el mayor número de personas, incluyendo a quienes se encuentran en con-

ACCESIBILIDAD*3 dición de discapacidad, y de igual forma, a aquellos que no cuentan con condiciones técnicas y tecnológicas adecuadas.

El curso tendrá una versión offline disponible en formato físico portable (CD- USB).

Propiedad que le permite a los recursosser modificados, ajustados o

De acuerdo con los organismos internacionales y en consideración con los aspectos particulares para el caso de la Universidad del Quindío, se elaboró una definición de estándar para la institución: este se concibe como un conjunto de especificaciones técnicas, pedagógicas y administrativas que regulan el diseño, la producción y la implementación de los recursos educativos, cuyo objetivo es la interoperabilidad entre dichos recursos construidos por diferentes profesores, en diferentes programas, con variedad de soportes y bajo criterios de unificación, normalización y racionalidad del costo. Dichas especificaciones poseen características abiertas que propician la construcción colaborativa de recursos educativos, entendidos estos en una acepción amplia que abarca desde la dimensión más granular de un recurso educativo -los objetos de aprendizaje- hasta la más compleja - las plataformas LMS-.

\section{Especificaciones para el estándar}

Se presenta para esta investigación, un conjunto de especificacionesde acuerdo a particularidades detec-

ADAPTABILIDAD *

USABILIDAD *

PEDAGÓGICO

\section{COMUNICATIVO}


CLASIFICABLE Propiedad que posibilita la catalogación y ordenamiento de contenidos y cursos.

LICENCIADO

Característica que define las condiciones de uso delos recursos.

*Las definiciones de las especificaciones marcadas con asterisco son tomadas de las características de los Recursos Educativos Digitales Abiertos (REDA), definidos en el Documento REDA del MEN.

\section{Configuración general de los cursos}

A partir de la implementación de estas especificaciones, los en las Plataformas, deberán seguir las siguientes recomendaciones para la configuración general del curso:

Nombre del curso o espa-
cio formativo

Nombre corto
Debe reflejar el contenido general del curso, éste debe ser claro y conciso.

Debe contener mayúscula inicial en cada palabra, excepto en las preposiciones, las siglas deben estar en mayúsculas. Ejemplo:Estrategias para Desempeño en AVA Cohorte IX

Debe contener los siguientes elementos:

1) Las iniciales de la Facultad, dispuestas así:

- Ciencias Humanas y Bellas Artes: $\quad \mathrm{CH}$

- Educación: ED

- Ciencias de la Salud: $\quad$ CS

- Ingeniería: IN

- Ciencias Económicas y Administrativas: CE

- Ciencias Agroindustriales: CA

- Ciencias Básicas y Tecnológicas: $\quad \mathrm{CB}$

2) Año, del cual se tomaron los dos últimos dígitos.

3) Semestre (entendido como el período académico del año), que se codificará con los números 1 o 2 , según corresponda.

4) Las iniciales del nombre del curso

Programa académico Nombre del programa académico o dependencia administrativa a la cual pertenece el curso o espacio formativo.

Descripción general del Descripción del curso en 140 caracteres. El texto debe contener una síntesis sobre los curso aspectos fundamentales del curso, tales como: temas, competencias a desarrollar (Usar mayúscula inicial).

Unidades Número total de unidades, módulos y secciones que conforman el curso.

\section{Aspectos Comunicativos}

La comunicación se considera un componente de gran importancia en la calidad del curso, por ello se considera que todo curso debe considerar los siguientes aspectos.

El curso debe contener un video de presentación del docente, quien rápidamente hace Presentación del docente una presentación personal y profesional. Este video puede estar integrado con el video de presentación del curso.

Esta presentación puede hacerse a través de video o de audio. En este recurso, el docente

Presentación del curso hace una síntesis o introducción que permita al estudiante conocer las generalidades del curso (contenidos. evaluación, metodología, horarios, cronograma, etc.).

Medios de asesoría do- El docente debe explicitar los medios a través de los cuales hará asesorías y seguimiento cente a los estudiantes, los horarios de atención y lugares de encuentro. 
Tutoriales y video tuto-
riales

Tiempo de respuesta

Foro dudas e inquietudes

Foro conozcámonos

(Novedades)

Perfil

Calendario
Sí se requiere el docente debe facilitar las mediaciones necesarias para que los estudiantes conozcan cómo utilizar equipos y aparatos dentro de las diferentes prácticas y laboratorios.

El tiempo de respuesta a las consultas e inquietudes de los estudiantes del curso ha de darse en un tiempo no mayor a 24 horas.

Este espacio de participación le ofrece la oportunidad de consignar las inquietudes de interés común sobre los contenidos y actividades del módulo. Este foro debe proponerse como un espacio colaborativo, donde se encuentran respuestas y orientaciones, no sólo por parte del profesor, sino también de los demás compañeros integrantes del grupo.

Este espacio permitirá a los estudiantes, conocerse e interactuar con sus compañeros de curso.

Estudiantes: datos de contacto, fotografía, pregrado que estudia, intereses académicos, culturales y deportivos.

Docentes: datos de contacto, fotografía, intereses académicos, culturales y deportivos, formación profesional y experiencia laboral.

Este recurso relaciona el desarrollo de las actividades del curso, mostrándolas en el bloque de eventos próximos.

\section{Aspectos pedagógicos}

Dada la magnitud, el componente más complejo, en la creación de cursos, es el uso contundente de argumentos pedagógicos que contribuyan al logro de los objetivos trazados de manera eficiente, pues depende de muchos factores e involucra en gran medida las actividades del docente en el desarrollo día a día de los cursos. Un curso estratégicamente diseñado, con un componente pedagógico definido, se espera que sea un curso exitoso, pero siempre está en función del profesor que oriente el curso. Sin embargo se proponen como aporte al componente pedagógico, las especificaciones que a continuación se citan.

Guía General

Metodología

Red conceptual

\section{Producción Intelectual propia}

Como parte del desarrollo del curso virtual, es necesario que el docente, elabore una guía general del curso disponible en plataforma.

Con base en los créditos académicos o la intensidad horaria del curso virtual, así como en las relaciones entre tiempo de estudio independiente del estudiante (TI), el tiempo para el trabajo colaborativo entre estudiantes (TC) y el tiempo de trabajo de tutoría o acompañamiento por parte del docente-tutor (TT), el docente debe proponer un cronograma de trabajo que permita al estudiante organizar sus tiempos de estudio diario o semanal, especificando cuáles de las actividades de aprendizaje propuestas serán retroalimentadas y calificadas, así como su porcentaje frente a la calificación final. Esto implica cuantificar el tiempo que debe dedicar el estudiante al estudio del material de lecturas, al abordaje de actividades, a la búsqueda de información o trabajo en equipo, a la preparación de los aportes académicos, y demás desempeños en que deberá incurrir el estudiante bajo los principios de autodisciplina y aprendizaje colaborativo, precisando también los tiempos en los que acompañará o recibirá retroalimentación del tutor.

Muestra un esquema o mapa de conceptos propios del curso.

Se debe priorizar la elaboración de contenidos propios que expresen las ideas, pensamientos y criterios de los docentes, frente a otras fuentes y otros autores. Esto, con miras a ampliar el repositorio de recursos educativos disponibles para el desarrollo de las actividades de aprendizaje. 
Evaluación

Recursos educativos

Bibliografía / webgrafía
Se deben acoger las recomendaciones sobre la evaluación del aprendizaje, adoptada por la Universidad en el respectivo sílabo. Para el diseño, montaje y desarrollo de cursos, se debe explicitar la calificación y la evaluación.

Además se deben especificar claramente los porcentajes de cada uno de los componentes de la evaluación.

En los foros se evaluará la calidad de las intervenciones allí consignadas.

Todos los libros, lecturas, recursos y material complementario para el logro de los objetivos deben ser facilitados, respetando los derechos de autor.

El docente debe aportar todos los vínculos o enlaces de acceso al material de estudio (e. g. sitios, bibliotecas digitales o repositorios de contenidos educativos) teniendo en cuenta derechos de autor y licencias de uso.

\section{CONCLUSIONES}

- Se hizo una caracterización de los procesos virtuales en la Universidad. De allí surgió la importancia de considerar componentes esenciales al implementar estrategias en la creación de cursos, tales como: el respeto por los derechos de autor y aspectos académico-administrativos.

- A través del levantamiento de información se constató que, usabilidad, accesibilidad, adaptabilidad y modularidad son conceptos validados y compartidos a nivel internacional, presentados por diferentes entes especializados en estandarización. En consonancia, transversalidad, transmedialidad, diseño funcional, gestión administrativa, licenciamiento y unidad conceptual son conceptos que responden a reflexiones educativas contextuales.

- Se diseño un conjunto de especificaciones técnicas, administrativas y pedagógicas que condujeron al establecimiento de un manual de buenas prácticas, que pueda evolucionar hacia un estándar E-learning, que reúna los lineamientos de las organizaciones internacionales y se fortalezca con las necesidades y proyecciones locales.

- Para que una experiencia E-learning sea efectiva es necesario que los lineamientos pedagógicos, comunicativos, organizacionales y tecnológicos de su diseño guarden coherencia entre sí. Aunque los estándares están construidos para cumplir normas, en este ejercicio de reflexión e investigación se ha comprendido la necesidad de acomodar especificaciones generales a situaciones contextuales. Habida cuenta de que la compleja realidad educativa debe abordarse desde un pensamiento flexible, los estándares se conciben como especificaciones orientadoras para el diseño de procesos educativos que incorporan lo virtual y lo tecnológico.

- La creación de un espacio virtual debe ser pensado y diseñado desde una perspectiva que permita distribuir y compartir el conocimiento. Así, la incorporación de estándares en educación debe propender a dar claridad en los procedimientos que deberían tenerse en cuenta en la producción e implementación. La normalización debe aplicarse con el propósito de establecer procesos y procedimientos que articulen y hagan evidente en cada diseño el diálogo coherente de los lineamientos que constituyen la Estrategia Virtual. 


\section{BIBLIOGRAFÍA}

- $\quad$ Agudelo B., N. M. (Marzo de 2013). Colombia Aprende. Consultado en Marzo de 2014. Ministerio de Educación Nacional: aprendeenlinea.udea.edu.co/lms/men/docsoac3/0301_metadatos.pdf.

- Carmona S., E. J., (2013). Hacia la Sociedad del Conocimiento. Revista de investigaciones Universidad del Quindio. Vol 2, Nro 24. Septiembre de 2013.

- Carmona S, E. J., \&Gallego Berrío, L. M. (2012). Procesos educativos mediados por plataformas virtuales en la metodología presencial usando b-learning.Revista de investigaciones Universidad del Quindio. Vol 2, Nro 23. Diciembre de 2012.

- Carrasco Vargas, S., \& González Escobar, V. (2011). Concepción de E-learningen el contexto del país: Un uevo concepto de mundo, de empresa y de educación. En C. Rama, \& J. Domínguez Granada, El aseguramiento de la calidad de la educación Virtual. Chimbote, Perú: Gráfica Real.

- Facundo, A. (2011). Análisis del aseguramiento de la calidad de la educación superior. En C. Ramos, \& J. Domínguez Granada, El aseguramiento de la calidad de la educación virtual. Chimbote, Perú: Gráfica Real.

- Fernández-Manjón, B., Moreno-Ger, P., Sierra, J. L. y Martínez-Ortiz, I. (2008). Uso de estándares aplicados a TIC en Educación. Informe $N^{o} 16$. CNICE.

- Hilera González, J. R., \& Hoya Marín, R. (2010). Estandares ELearning: Guía de consulta. Alcalá, España: Universidad de Alcalá.

- Hilera González J.R. (2013). Guía metodológica para la implantación de desarrollos curriculares virtuales accesibles. Universidad de Alcalá, Madrid.

- $\quad$ ISO. (2009). International Organization for Standarization. Obtenido de www.iso.org

- Ministerio de Educación Nacional, MEN. (2012). Recursos Educativos Abiertos. Bogotá: Colombia: Graficando Servicios Integrados.

- Morales Saldarriaga, J. C. (2014).Estado de la legislación colombiana para la creación de programas profesionales virtuales con un alto componente de accesibilidad. Institución Universitaria CEIPA. Libro de actas CAFVIR2014. V congreso internacional sobre calidad y accesibilidad de la Formación virtual. Pág. 133-140.

- Sierra, H. (2009). Modelo pedagógico Humanista tecnológico de la Dirección Nacional de Servicios Académicos Virtuales UN Virtual. Obtenido de www.virtual.unal.edu.co/ unv/portal/articles.

- Varela, C. (2015). Formación virtual basada en calidad, innovación y accesibilidad. Universidad de Asunción Paraguay. Libro de actas CAFVIR2015. Formación virtual inclusiva y de calidad para el siglo XXI. Pág. 26-31.

- Viscay, C. E. (2005). Los estándares de E-learning. (U. d. Virtual, Ed.) Ciencia y Tecnología. 